\title{
Measurements of cosmic-ray secondary nuclei at high energies with the first flight of the CREAM balloon-borne experiment
}

\author{
H.S. Ahn ${ }^{\text {a }}$, P.S. Allison ${ }^{\text {b }}$, M.G. Bagliesi ${ }^{\text {c }}$, J.J. Beatty ${ }^{\mathrm{b}}$, \\ G. Bigongiari ${ }^{\text {c }}$, P.J. Boyle ${ }^{\mathrm{d}}$, T.J. Brandt ${ }^{\mathrm{b}}$, J.T. Childers ${ }^{\mathrm{e}}$, \\ N.B. Conklin ${ }^{f}$, S. Coutu ${ }^{f}$, M.A. Duvernois ${ }^{e, 1}$, O. Ganel ${ }^{\text {a }}$, \\ J.H. Han ${ }^{g}$, H. J. Hyun ${ }^{g}$, J.A. Jeon ${ }^{g}$, K.C. Kim ${ }^{\text {a }}$, J.K. Lee ${ }^{\mathrm{g}}$, \\ M.H. Lee ${ }^{\text {a }}$, L. Lutz ${ }^{\text {a }}$, P. Maestro ${ }^{c}$, A. Malinin ${ }^{\text {a }}$, \\ P.S. Marrocchesi ${ }^{\text {c }}$, S.A. Minnick ${ }^{\text {h }}$, S.I. Mognet ${ }^{\mathrm{f}}$, S. Nam ${ }^{\mathrm{g}}$, \\ S.L. Nutter ${ }^{\mathrm{i}}$, I.H. Park ${ }^{\mathrm{g}}$, N.H. Park ${ }^{\mathrm{g}}$, E.S. Seo ${ }^{\mathrm{a}}$, R. Sina ${ }^{\mathrm{a}}$, \\ S.P. Swordy ${ }^{\text {d,* }}$, S.P. Wakely ${ }^{d}$, J. Wu ${ }^{a}$, J. Yang ${ }^{g}$, Y.S. Yoon ${ }^{a}$, \\ R. Zei ${ }^{c}$, S.Y. Zinn ${ }^{\text {a }}$ \\ ${ }^{a}$ Institute for Physical Science and Tech., University of Maryland, College Park, \\ MD 20742, USA \\ ${ }^{\mathrm{b}}$ Department of Physics, Ohio State University, Columbus, OH 43210, USA \\ ${ }^{\mathrm{c}}$ Department of Physics, University of Siena \& INFN, Via Roma 56, 53100 Siena, \\ Italy \\ ${ }^{\mathrm{d}}$ Enrico Fermi Institute and Dept. of Physics, University of Chicago, Chicago, IL \\ 60637, USA \\ ${ }^{\mathrm{e}}$ School of Physics and Astronomy, University of Minnesota, Minneapolis, MN \\ 55455, USA \\ f Pennsylvania State University, University Park, PA 16802, USA \\ ${ }^{g}$ Department of Physics, Ewha Womans University, Seoul 120-750, Republic of \\ Korea \\ ${ }^{\mathrm{h}}$ Department of Physics, Kent State University, Tuscarawas, New Philadelphia, \\ $\mathrm{OH} 44663$, USA \\ ${ }^{\mathrm{i}}$ Department of Physics, Northern Kentucky University, Highland Height, KY \\ 41099, USA
}

\begin{abstract}
We present new measurements of heavy cosmic-ray nuclei at high energies performed during the first flight of the balloon-borne cosmic-ray experiment CREAM (Cosmic-Ray Energetics And Mass). This instrument uses multiple charge detectors
\end{abstract}


and a transition radiation detector to provide the first high accuracy measurements of the relative abundances of elements from boron to oxygen up to energies around $1 \mathrm{TeV} / \mathrm{n}$. The data agree with previous measurements at lower energies and show a relatively steep decline $\left(\sim E^{-0.6}\right.$ to $\left.E^{-0.5}\right)$ at high energies. They further show the source abundance of nitrogen relative to oxygen is $\sim 10 \%$ in the $\mathrm{TeV} / \mathrm{n}$ region.

Key words: cosmic rays, cosmic-ray propagation, PACS: 96.50.S

\section{Introduction}

The first flight of the Cosmic-Ray Energetics and Mass (CREAM) highaltitude balloon experiment took place in Antarctica during December 2004 and January 2005. A central goal of this flight was to investigate the source properties of cosmic rays through the careful measurement of those cosmic nuclei which are predominantly of secondary origin.

The escape of particles from the Galaxy during propagation is known to be energy dependent at high energy. As a result, the spectrum of cosmic rays measured at Earth is different from the spectrum of these particles at their source. Understanding the magnitude of this difference is crucial for solving the puzzle of cosmic-ray origins. Secondary nuclei are particularly useful in addressing this goal because they are produced largely by the spallation interactions of primary particles as they propagate from their source regions through the interstellar medium to Earth. The pattern of relative abundances of secondary and primary nuclei therefore encodes information about the propagation history of the primary cosmic rays, allowing us to identify the contribution from propagation to the spectrum observed at earth. A recent review has identified the measurement of secondary nuclei abundances as a key goal for achieving future progress in cosmic-ray science [1].

It is known from earlier measurements (see, e.g., 223]4/5]) that the diffusion escape time for particles from our Galaxy decreases with increasing particle energy, or magnetic rigidity. This decrease can be described as a power law above some threshold rigidity near 2-10 GV. Below this rigidity, the escape time is roughly constant and the interstellar pathlength is approximately proportional to the particle velocity $\beta(=\mathrm{v} / \mathrm{c})[6]$. Above $10 \mathrm{GV}$, the escape time can be characterized as a pathlength (in $\mathrm{g} / \mathrm{cm}^{2}$ ) by assuming a constant density for the interstellar medium. A typical form for the rigidity dependence of

\footnotetext{
* Corresponding author. Address: s-swordy@uchicago.edu

1 now at the University of Hawaii
} 
this quantity is $\lambda=\lambda_{0}\left(R / R_{0}\right)^{-\delta}$. Here $\lambda$ is the mean escape pathlength, $R$ is the nucleus magnetic rigidity, and $\lambda_{0}\left(\sim 10 \mathrm{~g} / \mathrm{cm}^{2}\right)$ is the pathlength at the threshold rigidity $R_{0}$ (typically $10 \mathrm{GV}$ ).

The variable $\delta$ is the key parameter in the description of cosmic-ray diffusion from the Galaxy at high energies. In the simplest models of cosmic-ray transport, primary particles from a source with spectral index $\alpha$ escape more easily at high energy to produce an steepening in the slope of the energy spectrum observed at Earth to an index of $\alpha+\delta$.

The propagation of cosmic rays in our Galaxy is an essentially statistical process at energies where the radius of gyration in typical bulk fields is significantly less than the size of randomness in the magnetic field structures. In practice, this means that at the energies where the measurements of secondary nuclei have thus far been possible $(<100 \mathrm{GeV} / \mathrm{n})$ the propagation is indeed well-approximated by models where cosmic rays simply diffuse out of the Galaxy. As a result, the pathlength distribution for each individual nuclear species is close to an exponential in form, with a mean value at high energy given by the expression above.

The standard procedure when measuring secondary nuclei is to compare their abundances to those of their most likely (primary) parent nuclei, and examine how these ratios vary with energy. This can determine the value of the escape energy dependence, $\delta$. For example, the ratio of boron (essentially all of which is secondary) to its most likely parent nucleus, carbon, is expected to decline with energy with a slope which almost directly yields a value for $\delta$ above $\sim 30$ $\mathrm{GeV} / \mathrm{n}$. nitrogen, on the other hand, is only partly secondary; it is known to have a residual abundance at the source. The ratio of nitrogen to oxygen is expected to decline less rapidly with energy. Finally, the ratio of the two primary nuclei carbon and oxygen is expected to remain relatively constant with energy.

In this work, we describe measurements of the ratios of boron to carbon, nitrogen to oxygen, and carbon to oxygen over an energy range of $1 \mathrm{GeV} / \mathrm{n}$ $1 \mathrm{TeV} / \mathrm{n}$. Heavier nuclei $(\mathrm{Z}>8)$ will be the subject of future publications. One crucial aspect of these measurements is the correction for secondary particle production in the local atmospheric overburden above the balloon instrument. The systematic error associated with this correction becomes significant at high energies and ultimately imposes a limit for the maximum energy at which such measurements might be pursued on a balloon flight. 


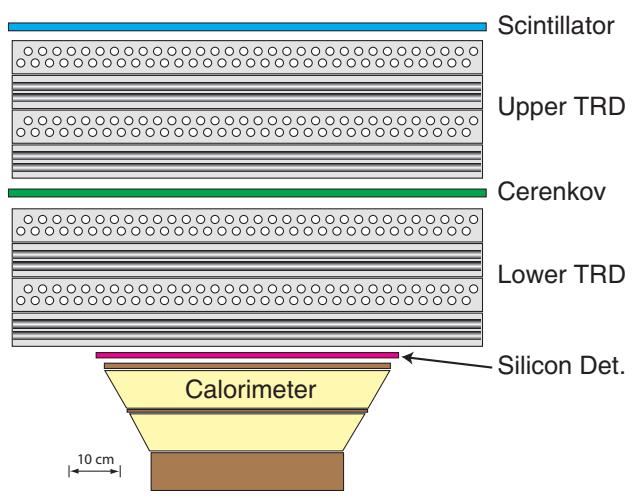

Fig. 1. A Schematic view of the CREAM instrument for the first flight.

\section{CREAM Instrument and Flight}

The CREAM instrument was first launched from Williams Field near McMurdo Station in Antarctica on December 16th, 2004. The payload circled the South Pole three times in a flight which lasted nearly 42 days[7]. The mean altitude during the flight was $38.6 \mathrm{~km}$, corresponding to an average atmospheric overburden of $3.9 \mathrm{~g} / \mathrm{cm}^{2}$. The data for the present study were provided by a trigger scheme designed to tag heavy nucleus events (charge $\mathrm{Z} \gtrsim 3$ ). We term these triggers "Hi-Z" events, around 15 million of these events are used in the present analysis.

The instrument configuration used for this flight is shown schematically in Figure 1. A much more detailed description of the instrument, along with a discussion of calibration processes, detector performance, and general architecture is given in a recent instrument paper [8]. Here we briefly review only those detector elements used for the Hi-Z analysis.

The detector system consists of a stack of horizontal components resting on a solid base plate. Importantly, there is no pressure gondola shell for CREAM. Not only does this significantly decrease the mass of the payload, it reduces the material overburden of the instrument, limiting the probability of local interactions of cosmic-ray nuclei. Such interactions can cause backgrounds of secondary particles via charge-changing nuclear processes.

At the base of the detector stack is a hadronic calorimeter (CAL) which has been described in detail elsewhere [9]. The calorimeter is designed to measure particles at the highest energies and is not used in the present analysis. Above the CAL is a silicon charge detector (SCD) consisting of 2912 individual pixels, each with an area of $1.55 \times 1.37 \mathrm{~cm}^{2}[10$. The pixels overlap slightly to provide full coverage over a total area of $78 \times 79 \mathrm{~cm}^{2}$. The dynamic range of the readout system can accommodate nuclei from $Z=1$ to $Z=28$. Over this range the detectors have a remarkably linear response. 
Above the SCD lies a precision gas transition-radiation detector (TRD)[11], split into two segments. Each segment consists of blocks of plastic foam $(120 \times$ $120 \mathrm{~cm}^{2}$ in area) in which are embedded $2 \mathrm{~cm}$ diameter thin-walled proportional tubes. There are 256 such tubes in a segment, each filled with a $95 \%$ xenon $5 \%$ methane gas mixture. The CREAM coordinate system is defined with the $z$ direction vertical in Figure 1, with perpendicular $x$ and $y$ directions corresponding to the major axes of the detector planes. The TRD tubes in each segment are divided into 4 sets of 64 , each of which is alternatively aligned with either the $x$ or $y$ axis. This provides a view of the particle track in both the $x z$ plane and the $y z$ plane.

Each proportional tube in the array is instrumented with a dual-gain amplifier system which provides a dynamic range of $\sim 4000$. This allows for the measurement of nuclei from $Z=3$ to $Z=26$. Between the two TRD segments is an acrylic Cherenkov detector (CD), which is instrumented by eight photomultiplier tubes (PMTs) viewing wave-shifter bars along the edges of the plastic. The central radiator has a refractive index near 1.5 and is doped with a wave-shifting dye to provide near-isotropic emission within the volume.

At the top of the instrument is a plastic scintillation assembly, the Timing Charge Detector (TCD). The detector consists of eight $120 \times 30 \times 0.5 \mathrm{~cm}^{3}$ paddles, viewed at each end by fast photomultipliers. They are arranged in two orthogonal layers of four paddles each, providing a coverage area of $120 \times 120$ $\mathrm{cm}^{2}$. Both the timing and amplitude of the scintillator signals from each event are digitized by these detectors. In this work, however, we only use the event amplitudes. For these, the TCD has a dynamic range for nuclei from $Z=1$ to $Z=28$. The total grammage of the detector stack, from TCD to SCD is about $7.0 \mathrm{~g} / \mathrm{cm}^{2}$.

The nuclei presented in this paper are almost all in the energy range below $1 \mathrm{TeV} / \mathrm{n}$. The energy measurement device used in this regime is the TRD. Although the Calorimeter trigger threshold accepts events near the upper end of this energy regime, it is not included in the present analysis since it restricts the available collection aperture. While the amount of transition radiation produced at this energy is not large (the nominal threshold for production is around Lorentz factors $\gamma>10^{3}$ ), the proportional tube gas, xenon, has one of the largest known relativistic rises in ionization energy loss [12. This allows for an estimate of particle $\gamma$ in the range $4<\gamma<5 \times 10^{2}$. Importantly, the response of this detector has been calibrated during beam tests at CERN. This is discussed extensively elsewhere [13]. 

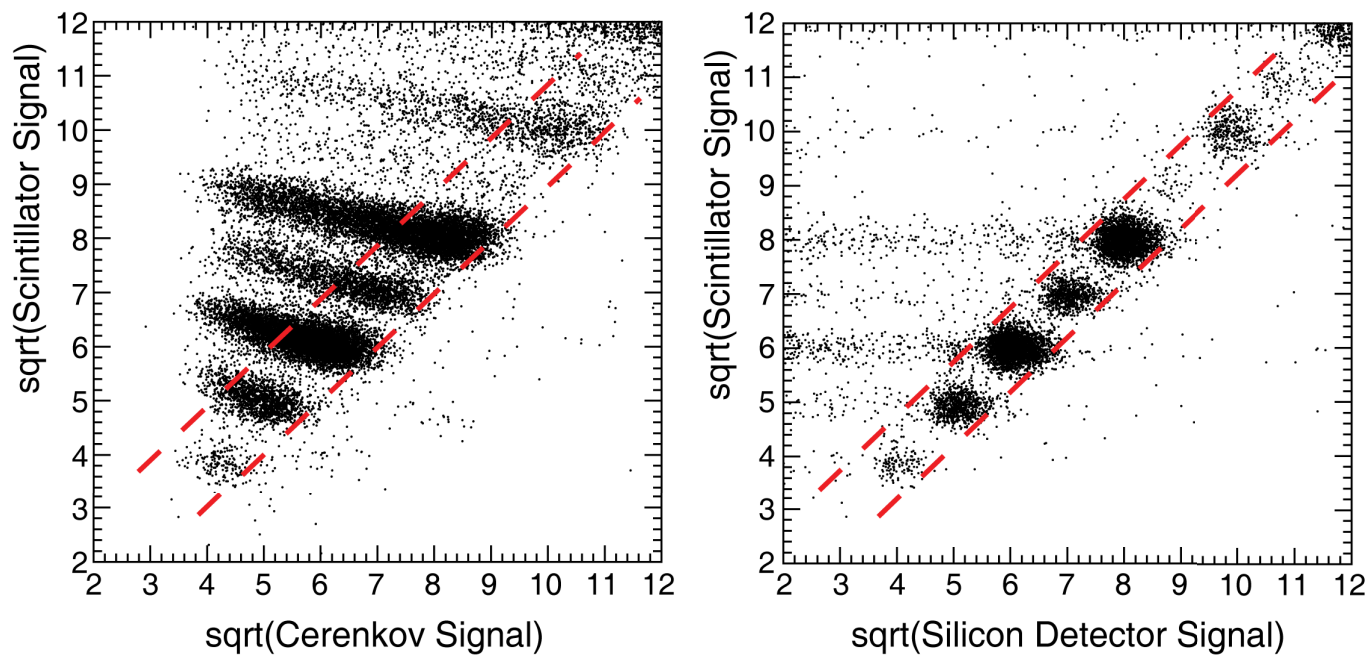

Fig. 2. Scatter plots of detector signals for a small fraction of the flight data. Left: The square root of the TCD signal versus the square root of the CD signal. Also shown are representative cuts applied to select relativistic nuclei (between dashed lines). The vertical scale here has been adjusted to approximate the nuclear charge and all signals are corrected for gain and pathlength. Right: The square root of the corrected TCD signal versus the square root of the corrected SCD for the selected relativistic nuclei from the left panel. Again, representative cuts are shown which select nuclei that do not undergo charge-changing interactions while traversing the instrument.

\section{Data Overview}

In this section we describe in some detail the data collected during the flight, as well as some of the corrections applied to those data. A Hi-Z event is required to generate a signal above a fixed threshold in both the CD and TCD within a coincidence window of $\sim 100 \mathrm{~ns}$. These thresholds were adjusted before the flight to ensure high efficiency for relativistic boron $(Z=5)$ nuclei, while rejecting the tail of the much more abundant Helium nuclei $(Z=2)$.

\subsection{Detector Corrections}

One of the benefits of a tracking instrument is that it provides a simple and direct mechanism for identifying and correcting non-uniformities in detector response. After constructing particle trajectories using the tracking algorithm described below, we apply mapping corrections for every event collected. The maximum level of these corrections is $\sim 50 \%$ for the TCD and $\sim 30 \%$ for the CD. No positional mapping is required for the silicon detector, but the individual pixels do receive a gain-matching correction. Again using tracking information, the signals from all the detector systems are also corrected for pathlength. 
In the TCD, an additional correction is made for the (Birk's Law) saturation in the scintillator and non-linearity of the PMT response. The TRD's proportional tube signals are also corrected for gain variations caused by fluctuations in pressure and temperature. These signals are gain-matched to a precision of $\sim 3 \%$ over the entire flight. Additionally, there were several calibration procedures implemented periodically throughout the flight, as discussed in [8].

\subsection{Charge Identification}

To outline the analysis procedures, we first discuss in general terms the identification of charges using the CREAM detectors. This initial estimate of charge is used as an input to one of the tracking algorithms. Later, in Section 4, we describe the full relativistic energy analysis which requires more detailed interpretation of the events. The left-hand panel of Figure 2 shows a small sample of events measured in the TCD and CD after applying the above corrections. The individual charges are clearly visible. The vertical TCD scale is arbitrary, but has been adjusted to approximate the derived charge of the incoming relativistic nuclei. Also apparent on this plot is the threshold setting for the CD (abscissa), which can be seen to admit relativistic boron nuclei, and with lesser efficiency, Beryllium.

In a polar flight, the geomagnetic cutoff rigidity is very low, admitting a large flux of low-energy particles. Since we are primarily interested in high-energy particles for this study, we use the Cherenkov Detector to identify and reject the low energy ones. The CD has a refractive index of 1.5, which provides a threshold velocity of $\beta \sim 0.7$. Particles with low CD signal are therefore easily identified as having an energy of a few hundred $\mathrm{MeV} / \mathrm{n}$, well below minimum ionization in the proportional tubes of the TRD. An example of the cuts used to reject such nuclei are shown in the left panel of Figure 2 (dashed lines).

Also shown in Figure 2 (right panel) is a scatter plot between the TCD and the SCD signals (after the above cuts are applied). In general, the charge measurements by these devices are in good agreement. However, there are a number of events in which the charge measured by the SCD (abscissa) is lower than that measured by the TCD. These are caused by nuclear interactions in the instrument. Such events represent a small fraction of the data[14] and can be easily removed from the data by a simple consistency check between the TCD and SCD signal. A representative cut is shown by the dashed lines on the figure.

After the relativistic particle cuts are applied to the CD data and the consistency cut has been applied between the TCD and SCD signals, the overall charge resolution can be determined. Figure 3 shows the mean of the SCD 

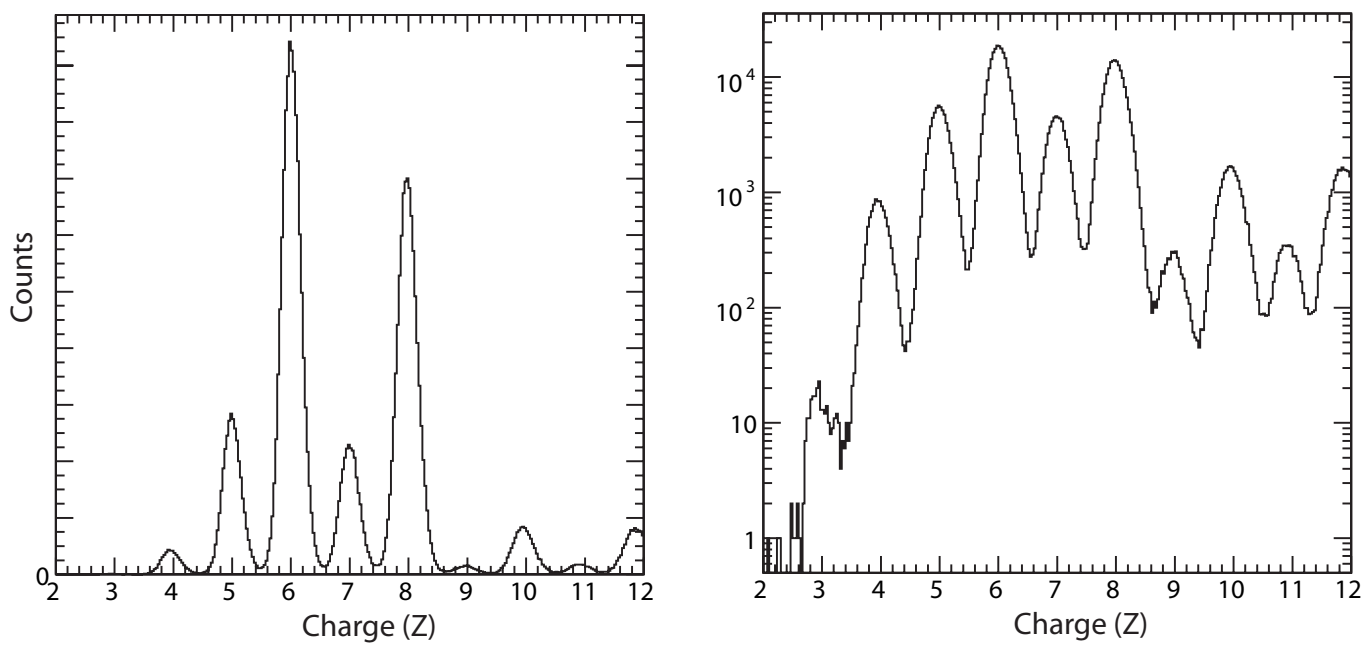

Fig. 3. Charge histogram of nuclei with Hi-Z triggers. The charge estimate shown is the mean of the square root of the corrected TCD signal and the square root of the corrected SCD signal. The data were selected using the cuts from Figure 2. The left-hand panel has a linear scale and the right-hand shows the same data with a logarithmic scale. The distribution for carbon has a width of $\sigma_{Z}=0.16$ charge units.

and TCD signals $((\sqrt{T C D}+\sqrt{S C D}) / 2)$ for relativistic Hi-Z, non-interacting particles collected during the flight. The charge distribution extends down to Lithium, which produces Hi-Z triggers at low efficiency because of the fixed CD threshold discussed above. Across the whole charge spectrum, all of the nuclei are visibly separated from their neighbors, including the rarer nuclei, Fluorine $(\mathrm{Z}=9)$ and Sodium $(\mathrm{Z}=11)$. Overall, the achieved charge resolution is excellent, with an RMS $(1 \sigma)$ resolution for carbon nuclei of $\Delta \mathrm{Z}=0.16$ charge units. Performance at this level is crucial for the successful study of rare secondary particles, as discussed below.

\section{Analysis Methods}

In the following section we will discuss the analysis procedures and corrections used to reduce the data from the CREAM flight. This includes a discussion of the final selection cuts (which are somewhat more restrictive than the cuts discussed above), the energy-determination mechanism, and energy deconvolution procedures. We begin with a synopsis of the track-fitting algorithms.

\subsection{Event Track Fitting}

The heart of the analysis is the track fit. The results of this fit are used in the mapping corrections described above to correct detector signals. They are also 
used to determine the actual track length of the particle in each of the TRD tubes it traverses. The accuracy of this information is crucial for properly determining the $d E / d x$ signal for each event. Because this number needs to be determined as well as possible, the fitted tracks must have a precision significantly less than a tube diameter. This is achieved by a multistage trackfitting procedure.

The first-stage track fitter employs a simple and very fast least-squares minimization which ignores the physical extent of the proportional tubes. Outlying signals are rejected from the fit by iteratively contracting an envelope around the estimated track position. Within 3 passes, the precision of this initial fit is $\sim 5 \mathrm{~mm}$ FWHM in both the $x y$ and $y z$ planes. The fit is then improved using a more sophisticated second-level algorithm which accounts for the full three-dimensional structure of the TRD. This algorithm uses the Minuit software package to perform a maximum-likelihood fit incorporating the different energy-loss distributions produced by tracks at different impact parameters. Because these distributions also vary with particle charge, an initial determination of particle charge $\mathrm{Z}$ (discussed above) is used as input to the algorithm. When initialized with the parameters provided by the level-one track, this fitter achieves an accuracy at the level of $\sim 1 \mathrm{~mm}$ RMS.

Using the tracking information, the TRD's $d E / d x$ signal is directly derived from the individual tube signals. A quality cut is applied which requires individual track segments to be at least $1.5 \mathrm{~cm}$ long, helping to minimize the impact of fluctuations for thin gas layers. A simple test of the accuracy of this process can be obtained by comparing the signal derived from the $x z$ plane to that of the $y z$ plane. These fits are essentially independent except for the common track energy-loss parameter, and therefore a comparison gives a rough estimate of the overall error in the determination of the $d E / d x$ value. The left panel of Figure 4 shows such a test for flight oxygen nuclei. The relative RMS difference between the $x z$ and $y z$ determinations is $\sim 14 \%$, implying that the resolution in the combined TRD is $\sigma \sim 7 \%$. The actual resolution, discussed below, is a little broader than this because of the correlations introduced by the track energy-loss parameter.

\subsection{Energy Determination}

The energy of incoming particles can be determined in two ways. At lower energies, the signal from the Cherenkov Detector can be used to estimate velocity, and hence energy. The TRD proportional tubes are used at energies above where the $\mathrm{CD}$ response has saturated. The signal in these tubes is produced by a combination of two energy-loss mechanisms, both of which scale with the energy of the nucleus. The primary component comes from Coulomb 

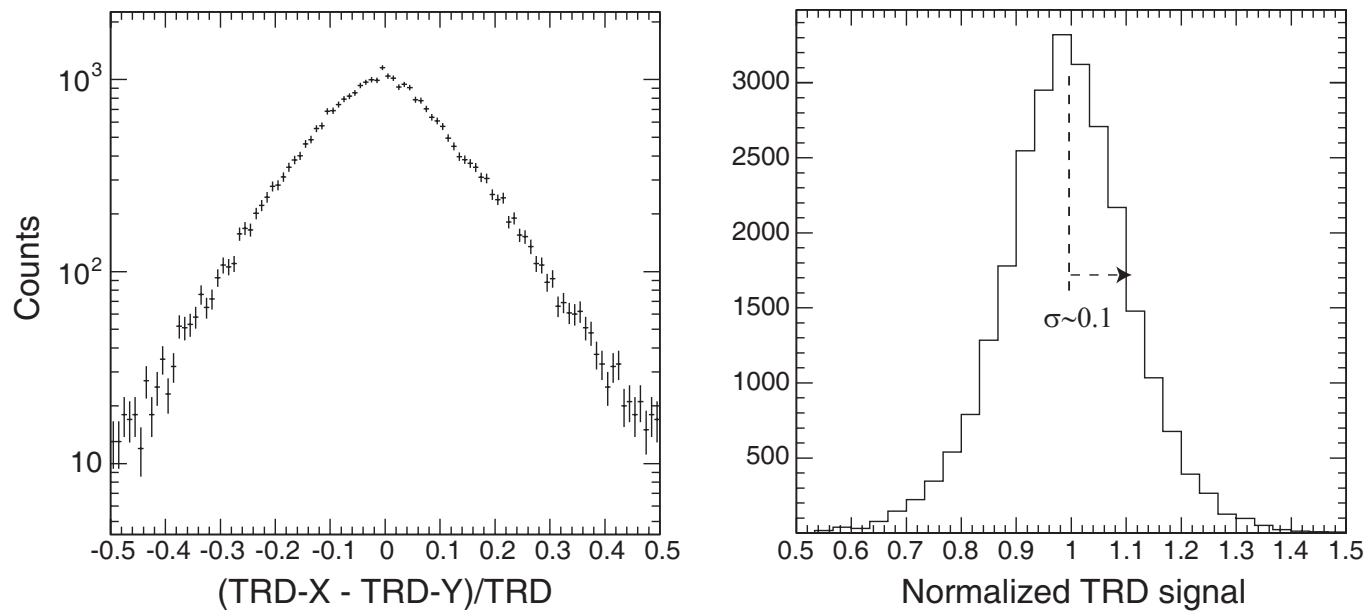

Fig. 4. Left: The fractional difference between the TRD signal determined from the $x z$ track and the $y z$ track for oxygen nuclei. The result has an RMS value of $\sim 14 \%$. Right: The TRD sum signal distribution for flight oxygen nuclei, selected to be at minimum ionization using the CD. The RMS resolution is $\sigma \sim 10 \%$. 
collisions of the nuclei with electrons in the gas. An additional contribution comes from any x-ray transition radiation absorbed in the xenon gas mixture. In the energy region of the present measurements $(\lesssim 1 \mathrm{TeV} / \mathrm{n})$, the tube signals are essentially all due to ionization energy loss.

The right panel of Figure 4 shows the distribution of TRD signals for oxygen nuclei which are selected to be close to minimum ionizing (using the Cherenkov detector). These events are also selected to have more than six TRD tubes with a usable signal. It is the width of this distribution (which remains essentially constant throughout the relativistic-rise region) that ultimately determines the energy resolution of the TRD. The RMS $(1 \sigma)$ width of the signal, S, distribution is $\Delta S \sim 8$ in these units. As demonstrated in Figure 5, which shows the response function of the TRD, this corresponds to a resolution in $\log _{10}(\gamma) \sim 0.3$, where $\gamma$ is the particle Lorentz Factor. This figure also shows the good agreement of the GEANT4[15] detector simulation (small points) with the results of a test beam calibration of the same configuration (large points). This calibration is discussed in more detail elsewhere [13,8], and has been shown to be consistent with the energies derived from the calorimeter signals in the $\sim 100 \mathrm{GeV} / \mathrm{n}$ region for carbon and oxygen 16$]$.

In practice, a simple least-squares energy fitter is used to determine the particle energy which best corresponds to the signals measured in the TCD, CD and TRD. For each event, a goodness-of-fit, $\chi_{E}^{2}$, is generated, based on the known response functions of these detectors.

\subsection{Selection Cuts}

The data for this study were selected from time periods when the experiment was relatively stable and when there was no recent gas venting, filling or other operations, and the atmospheric overburden was $\lesssim 5 \mathrm{~g} / \mathrm{cm}^{2}$. Periods were also avoided when the high voltage was being adjusted on any experimental system. These selections resulted in an effective data collection time of $\sim 21$ days.

The events used in the final analysis must pass various identification and quality cuts. The nuclear charge is selected by placing cuts on the TCD and SCD signal distributions, similar to those shown in Figure 2. An additional tracking cut helps here by requiring that the calculated particle trajectory pass through the SCD within $2 \mathrm{~cm}$ of the pixel with the largest signal. This cut keeps only "clean" events and eliminates possible albedo effects from the calorimeter just below the SCD. Approximately $70 \%$ of Hi-Z triggers with TRD data survive this cut. To provide adequate signal resolution, only events with more than six analog samples in the tube array are selected for analysis. In addition, the total track pathlength in the tubes is required to be at least 


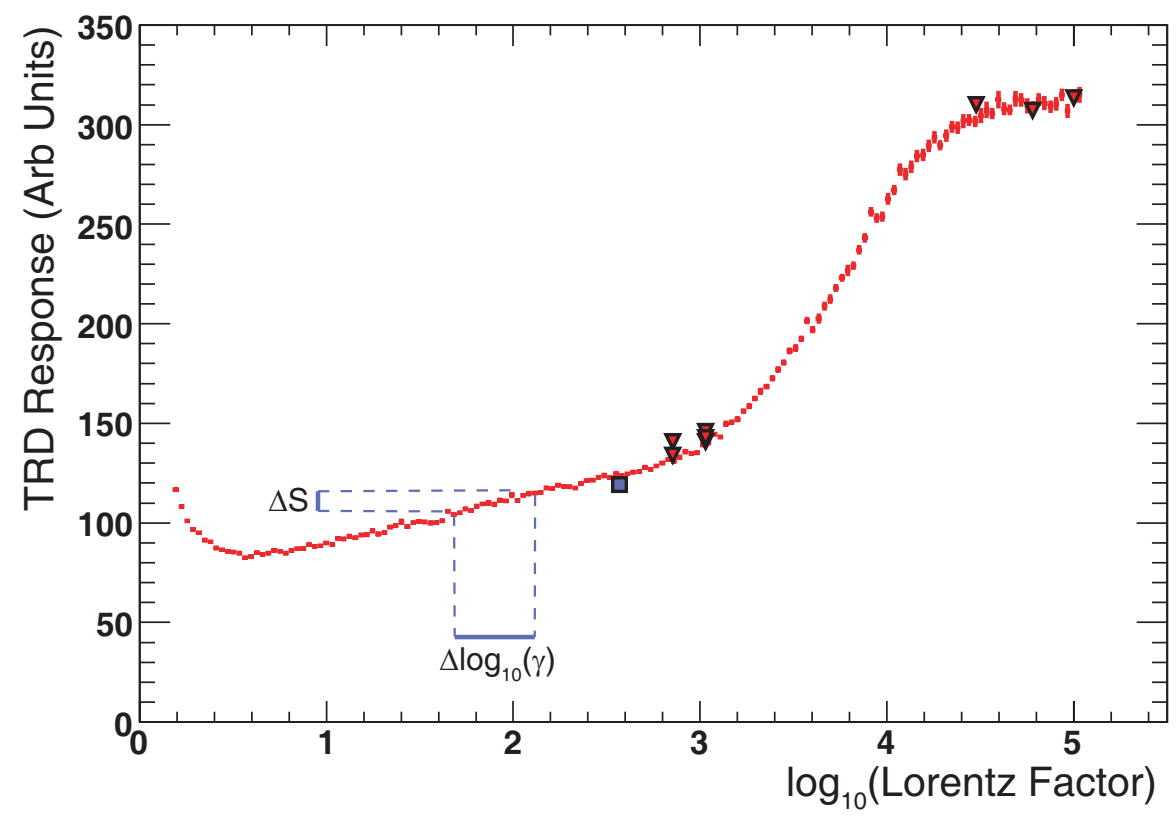

Fig. 5. The response of the TRD vs particle $\log$ (Lorentz factor). The small symbols are from a GEANT4 simulation of the detector configuration, and the large symbols are data from a direct accelerator measurement, as discussed in [13. Also shown schematically is the relationship between the TRD signal resolution and the $\log$ (Lorentz factor). 
$12 \mathrm{~cm}$. These two cuts admit $\sim 25 \%$ of the events with a good level-two track fit. A consistency check between the $x z$-plane TRD signal and the $y z$-view TRD signal (amounting to a simple cut on the distribution shown in Figure 4) further improves the overall resolution, while accepting $\sim 65 \%$ of the remaining events. A final cut is made on the likelihood of the track fit, rejecting poor geometry events, while keeping over $90 \%$ of those events which make it to this stage. The cumulative efficiency of all the cuts applied to the events which trigger the instrument in this analysis is $\sim 10 \%$.

As mentioned above, the energy fitter produces a simple $\chi_{E}^{2}$ value which can be used to reject pathological events. A loose selection cut is made that keeps only those events with reasonable values of $\chi_{E}^{2}<10$. This works rather well except when knock-on electrons, or other processes, produce non-statistical upwards fluctuations in the CD signals. This effect can cause a nucleus at low energy to mimic a high-energy event in the TRD. This is easily understood by referring to the TRD response curve shown in Figure 5. There is clear ambiguity in energy for a TRD signal of, for example, 115 scale units. The $\mathrm{CD}$ is needed to determine on which side of minimum ionization the event lies. A cut is therefore placed on the CD to ensure the events are on the high-energy side.

The flight data themselves can be used to estimate the effectiveness of this Cherenkov cut. In Figure 6 the CD signal is plotted for oxygen nuclei which are selected as discussed above except for the Cherenkov cut illustrated in Figure 2, These also are required to have a TRD signal between 30 and $35 \%$ above minimum ionization. According to Figure 5, these could either have an energy $\sim 400 \mathrm{MeV} / \mathrm{n}$ or $\sim 100 \mathrm{GeV} / \mathrm{n}$ from the response curve. It can be readily seen that there are two easily separable populations - corresponding to the low-energy and high-energy events. A simple cut on the CD signal (above 1.05 in this case) ensures only the high-energy particle survive.

\subsection{Charge Overlap Rejection}

Reliably discriminating between different charges is extremely important for any experiment measuring secondary nuclei. For example, because boron at high energy has only a few percent of the abundance of carbon, the peaks of the reconstructed populations of these nuclei must be at least three standard deviations apart to prevent a significant background from carbon from leaking into the boron sample.

With a TRD instrument, this requirement becomes even more stringent because the TRD signal response is not independent of $\mathrm{Z}$ (it scales as $\mathrm{Z}^{2}$ ). As a result, if a low-energy carbon nucleus gets misidentified by the charge detec- 

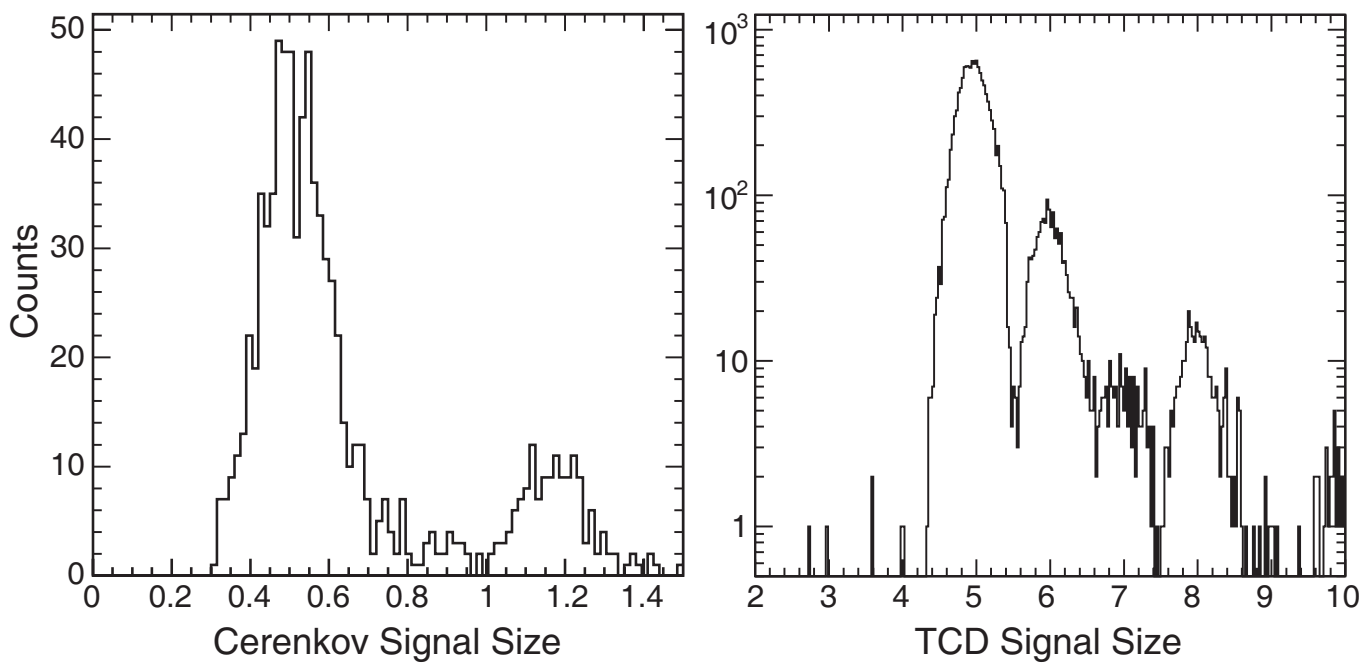

Fig. 6. Left: The distribution of Cerenkov signals from oxygen events selected to be between 115 and 125 on the response curve of Figure 5. See the text for details. The right-hand population of events comprises particles near $100 \mathrm{GeV} / \mathrm{n}$ and the left-hand peak is a tail of low-energy events near $400 \mathrm{MeV} / \mathrm{n}$ which have passed identical cuts. A cut at 1.05 provides effective discrimination between the two populations. Right: The distribution of charge signals measured by the TCD for events which have an SCD charge of $Z=5.0 \pm 0.2$. The effects of spallation are obvious as several of the nuclei enter the instrument with a charge larger than that measured in the SCD. 
tors as a boron, it will not be counted as a low-energy boron, but rather as a high-energy boron because of the incorrect $\mathrm{Z}^{2}$ normalization factor. This exacerbates the "charge overlap background" problem because the steeply falling cosmic-ray spectrum means there are thousands more low-energy carbon nuclei than high-energy boron nuclei.

Due to this additional effect, a separation of at least four standard deviations between the boron and carbon populations is required to make a backgroundfree determination of the boron abundance. A similar condition applies to the measurements of nitrogen and oxygen.

The CREAM instrument fortunately has two independent charge-determining detectors, the TCD and SCD, each of which has a resolution significantly less than 0.2 charge units (over the range of charges considered here). To combat the charge overlap problem, we place circular cuts of radius $\Delta \mathrm{Z}=0.2$ on all the charge peaks of the right panel of Figure 2, This selection produces effective cuts at $\Delta \mathrm{Z}= \pm 0.14$ on the summed charge distribution and completely removes interacting events. The resulting separation between the cut charge populations exceeds 4.5 standard deviations, thereby providing a clean sample of the low-abundance boron and nitrogen nuclei at high energies.

\subsection{Energy Deconvolution}

All the events passing the above cuts are collected into energy bins of width $\log _{10}(\mathrm{E})=0.4$. This corresponds to a width of $\sim 1.3 \sigma$ in terms of the energy resolution, discussed above. Approximately 45,000 relativistic carbon nuclei and 40,000 oxygen nuclei make it to this stage.

The true energy spectrum is derived from these binned values by correcting for energy spill-over between the bins due to the finite instrumental resolution. To account for these effects in a system where the resolution is a function of energy, a simulation is generally required. For this measurement, the primary energy determination device is the TRD, so we use an accurate simulation of the TRD and its fluctuations. This simulation uses a GEANT4 model of the upper CREAM payload including the TCD, TRD and SCD. The TRD component of this simulation has been discussed previously in [13].

The events from this simulation are passed through the same analysis code that is used to evaluate and fit the flight data. Various cross-checks are made to ensure the simulation provides an accurate representation of the data produced in the flight, including both mean signal levels and the fluctuations of those signals. Using the simulated results, a transfer matrix is obtained which can be used to deconvolve the measured energy bins to produce estimated true energy bin populations. This method, which has been discussed in previous 

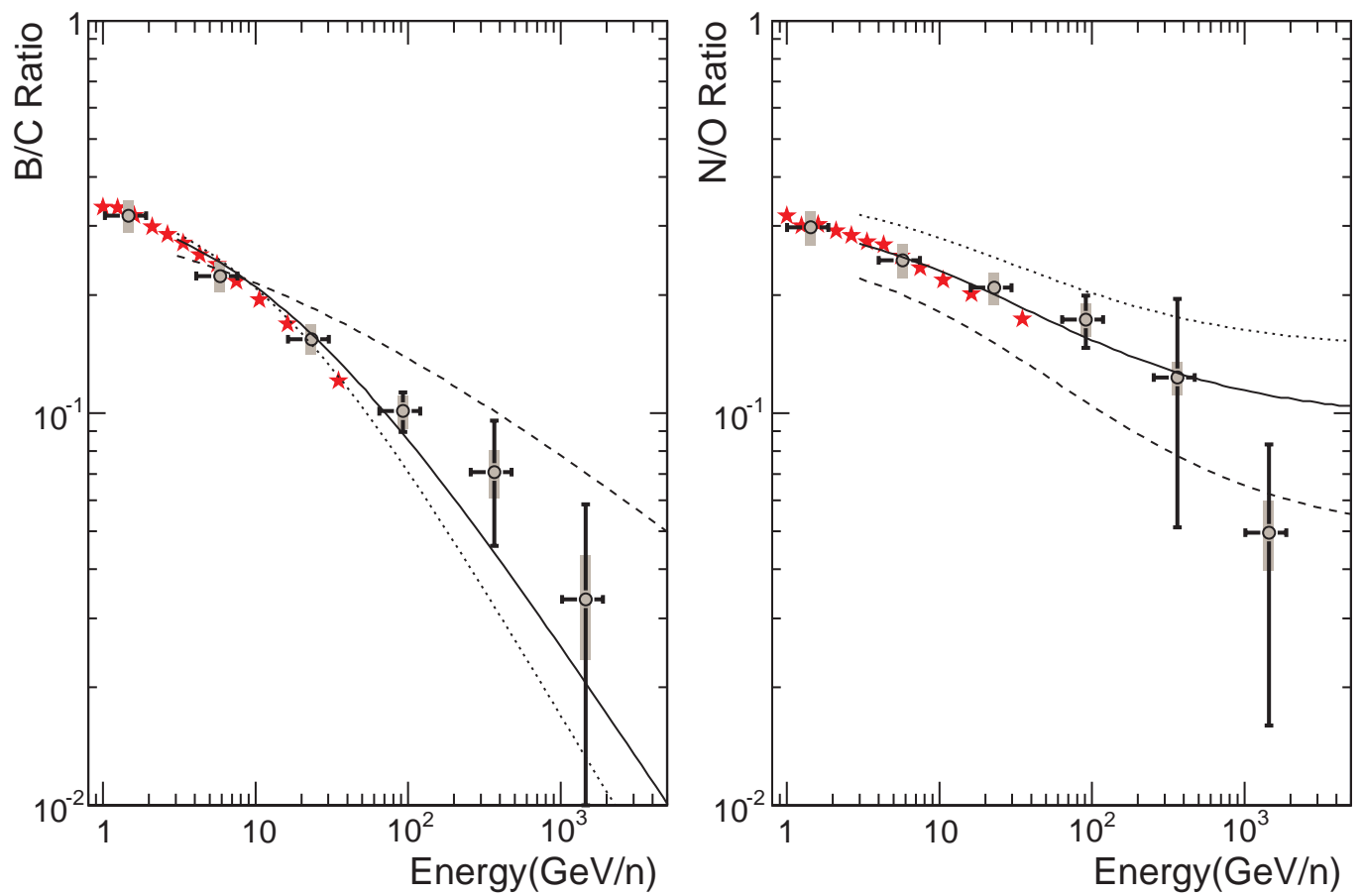

Fig. 7. Measurements of the ratios of nuclei as a function of energy. Left: Filled circles show the ratio of boron to carbon vs. energy after corrections. The horizontal errors are an estimate of the systematic error in the overall energy scale. The thin vertical lines correspond to the statistical error of the ratio and the grey bars show the systematic uncertainty in the ratio. See text for details. The lines represent model calculations for various values of the magnetic-rigidity dependence parameter, $\delta$, in escape from the Galaxy - as discussed in the text. These are; solid line $\delta=0.6$, long-dashed line $\delta=0.333$, short-dashed line $\delta=0.7$. The stars are data from the space experiment, HEAO-3-C2 [5]. Right: Filled circles show the ratio of nitrogen to oxygen vs. energy after corrections. The error bars and data points are as in the left panel. The lines represent model calculations of this ratio with the escape parameter $\delta=0.6$ (solid line in the top left-hand panel). The different curves correspond to different assumptions on the amount of nitrogen in the source material. These are; solid line source $\mathrm{N} / \mathrm{O}=10 \%$, long-dashed line source $\mathrm{N} / \mathrm{O}=5 \%$, short-dashed line $\mathrm{N} / \mathrm{O}=$ $15 \%$.

studies (e.g., [4]), is an iterative process since, for accurate results, the spectral index used in the simulation must match the (unknown) index of the data. However, as noted by several previous authors [17,18, the convergence of the iteration is rapid and small residual discrepancies have little impact on the final result. 

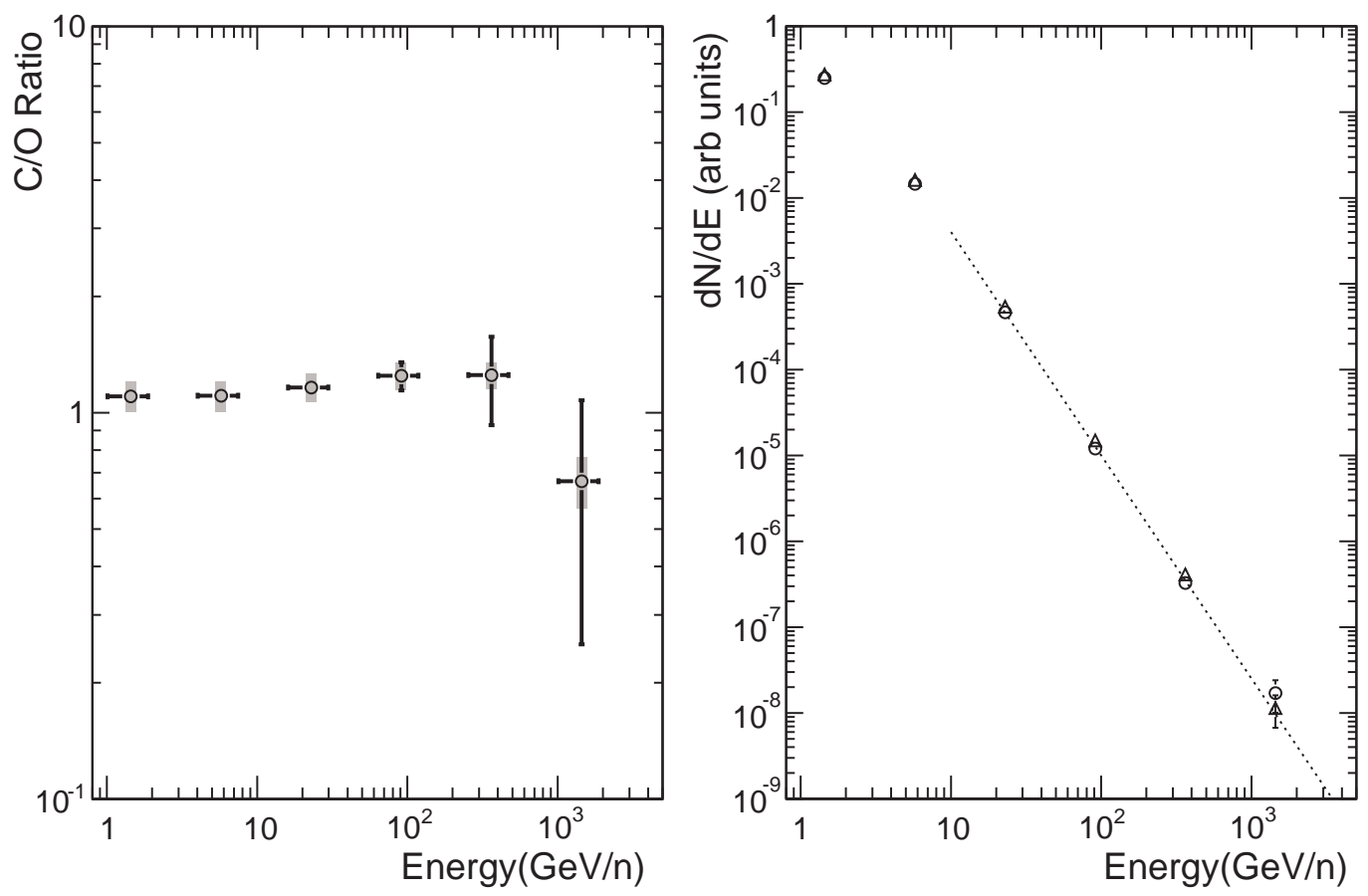

Fig. 8. Left: Filled circles show the ratio of carbon to oxygen vs energy after corrections. Right: The fluxes (energy $/ \mathrm{n} \times$ area $\times$ time $\times$ solid angle $)^{-1}$ (arb. units) of carbon nuclei (open triangles) and oxygen nuclei (open circles) from this measurement. The data are consistent with the dashed line, a power law $\propto(\text { Energy } / \mathrm{n})^{-\alpha}$ with a spectral index $\alpha=2.6$.

\section{Results and Corrections}

Using the analysis, selection cuts, and deconvolution scheme outlined in the previous sections, the flight data are processed to determine the ratios of elemental abundances as a function of energy into the $\mathrm{TeV} / \mathrm{n}$ region. As described above, this is achieved with a simple binning and deconvolution process which assigns the median energy of each bin by assuming a spectral index close to that of each denominator element. These results are given in Tables 1-3, and the associated Figures 7 and 8 . Also shown for comparison are the results from the space measurement HEAO-3-C2 [5] which used an assembly of Cherenkov counters of different thresholds to measure elemental composition up to $\sim 35 \mathrm{GeV} / \mathrm{n}$. These are the most statistically significant measurements to date in the lower energy region. These tables show the abundance ratios $\mathrm{B} / \mathrm{C}, \mathrm{N} / \mathrm{O}$, and $\mathrm{C} / \mathrm{O}$, respectively, as measured at the instrument as well as those values after extrapolation to the top of the atmosphere.

The corrections to the top of the atmosphere are made by considering separately the probability of charge-changing interactions in the instrument and the atmosphere. The right panel of Figure [6 clearly shows that events that have fragmented can be eliminated with charge cuts. However, the efficiency 
of these cuts must be accounted for in the analysis procedure.

Charge-changing interactions also occur in the atmosphere above the instrument. Obviously, these events cannot be identified, so a post-analysis correction must be applied using nuclear interaction models. For this purpose, we utilize both the partial cross-section model of Tsao et al. [19] and GEANT4 nuclear interaction simulations. These corrections become extremely important as the elemental abundance ratios decline. For example, if $3 \%$ of carbon nuclei $(\mathrm{Z}=6)$ change to boron $(\mathrm{Z}=5)$ in the atmosphere above our instrument this is a fairly negligible correction at $5 \mathrm{GeV} / \mathrm{n}$, where $\mathrm{B} / \mathrm{C} \sim 30 \%$. At around $\sim 1 \mathrm{TeV} / \mathrm{n}$, however, it becomes significant, since in this region $\mathrm{B} / \mathrm{C} \sim 5 \%$.

Since it appears that the $\mathrm{B} / \mathrm{C}$ ratio continues to decline relatively steeply with energy, the accurate determination of this ratio by balloon payloads will be intrinsically limited to the region below a few hundred $\mathrm{GeV} / \mathrm{n}$ because of uncertainties in these corrections. Measurements well above these energies can probably only be made successfully on a future space mission, where there are no systematic limitations from atmospheric corrections.

There are several different uncertainties documented in the tables. For the energy values, the quoted errors are systematic - arising from the precision with which the data and simulation response curves can be aligned. We estimate the fractional error in this process to be $\sim 3 \%$. In the response region where most of these data were collected, this precision corresponds to an energy-scale uncertainty of $\sim 15 \%$, as displayed in the tables.

The entries for the corrected abundance ratios feature both statistical and systematic errors. The statistical portion is derived in the standard way by propagating the statistical errors of each element in a ratio, using the original counts of events in the bins before deconvolution and before the atmospheric corrections. The systematic errors in each ratio stem from two effects. The first stems from residual uncertainty in the charge-dependent efficiency of the selection cuts used to construct the ratios. There are several cuts for which the charge dependence may amount to a few percent, so we use a conservative value of $10 \%$ for the total charge-dependent efficiency error. A second systematic effect derives from uncertainties in the atmospheric secondary corrections. The partial cross-section values in the literature for carbon+Air $\rightarrow$ boron in the relativistic region can differ by up to $\sim 30 \%$. The typical correction in the ratio of $\mathrm{B} / \mathrm{C}$ from atmospheric secondaries is of the order $\sim 3 \%$, and we assign a fixed $1 \%$ systematic error for the uncertainty in correcting the ratios to the top of the atmosphere. The systematic errors quoted in the tables reflect whichever contribution dominates in the appropriate energy region.

To provide a check on these measurements we also present data on the $\mathrm{C} / \mathrm{O}$ ratio in Figure 8 . As both $\mathrm{C}$ and $\mathrm{O}$ are primary nuclei - originating mostly in 
the cosmic-ray source - the expectation is that their abundance ratio should be close to constant with energy. This expectation is in agreement with our measurements.

A further check can be seen in the right panel in Figure 8. Here the measured intensities of carbon and oxygen nuclei are shown as a function of energy (these are differential elemental intensities given in arbitrary units since the overall live-time of the flight has not yet been accurately determined). Apart from demonstrating that the CREAM measurements reported here cover nearly 9 decades in differential intensity, these data show that the measured energy spectra for primary nuclei are consistent with a differential energy spectral index of $\alpha=2.6$ at energies above $10 \mathrm{GeV} / \mathrm{n}$. This value is near the average of previously-reported measurements and provides confidence that our analysis techniques and energy scales are accurate.

\section{Discussion}

The left panel of Figure 7 shows that the measured ratio of $\mathrm{B} / \mathrm{C}$ declines fairly steeply with energy into the $\mathrm{TeV} / \mathrm{n}$ region. Overlaid on this figure are three lines which represent the results of a simple leaky-box model of cosmic-ray propagation. For each of the lines, a different value of $\delta$ is used. The uppermost (long-dashed) line uses $\delta=0.33$, the solid line uses $\delta=0.6$, and the shortdashed line uses $\delta=0.7$. The small data points (stars) on the figure represent the ratio measurements of the HEAO-C2-3 experiment [5]. The present data are consistent with these measurements and also seem to be consistent with the $\delta=0.6$ curve.

Measurements of $\mathrm{N} / \mathrm{O}$ allow one to investigate the apparent source abundance of nitrogen in cosmic rays, a topic which has been discussed for many years. The cosmic nitrogen abundance is somewhat unusual in that it contains significant contributions from both primary and secondary sources. At a high-enough energy the primary component should come to dominate and the ratio N/O should flatten out. Our N/O measurements, again together with those of HEAO-C2-3 are shown in the right panel of Figure 7. As before, three simple leaky-box propagation models are overlaid on the data, in this case with the parameter $\delta$ fixed at 0.6 . The different lines on this plot correspond to different assumptions on the source abundance of nitrogen compared to oxygen. The solid line corresponds to a source $\mathrm{N} / \mathrm{O}=10 \%$, the long-dashed line corresponds to a source $\mathrm{N} / \mathrm{O}=5 \%$ and the short-dashed line is for source $\mathrm{N} / \mathrm{O}=15 \%$. Although lower-energy measurements on nitrogen isotopes have seemed to favor a source abundance $\mathrm{N} / \mathrm{O} \sim 5 \%(e . g ., 20]$, these new data, which agree well with $\mathrm{HEAO}[5]$, seem to prefer a source value $\sim 10 \%$. 


\section{Conclusions}

We have presented new measurements of the relative abundances of cosmic-ray secondary nuclei at high energies, where the energy dependence of cosmic-ray propagation is relatively unexplored experimentally. These results have been produced with the first flight of the CREAM high-altitude balloon instrument in Antarctica during austral summer 2004/2005. These are challenging measurements, and the results span over three decades in energy and over nine decades in particle intensity. The energy scale for these results is provided by a transition-radiation detector, operating mostly in the regime of the relativistic rise. A critical part of the success of this effort is the excellent resolution of the CREAM charge detectors. Both the TCD (plastic scintillator) and SCD (silicon detector) have demonstrated charge resolutions of $\Delta \mathrm{Z}<0.2$ charge units. This provides excellent charge isolation between primary nuclei and the much less abundant secondary nuclei at all energies. Indeed, without this level of charge separation, the background of misidentified nuclei would make this measurement difficult if not impossible above $\sim 100 \mathrm{GeV} / \mathrm{n}$.

The results presented here show the interstellar propagation pathlength decreases fairly rapidly with energy, with an energy dependence in the range $\delta \sim 0.5-0.6$. As a result, the propagation pathlength of cosmic nuclei is smaller by an order of magnitude for particles in the $\mathrm{TeV} / \mathrm{n}$ region compared to those at lower energies below $10 \mathrm{GeV} / \mathrm{n}$. This high-energy pathlength $\left(\sim 1 \mathrm{~g} / \mathrm{cm}^{2}\right)$, while small, is still large compared to the typical grammage of the Galactic disk $\left(\lesssim 0.002 \mathrm{~g} / \mathrm{cm}^{2}\right)$, and so it does not significantly constrain residual pathlength models proposed for higher energies[21] or in the source region[22]. The observations of $\mathrm{N} / \mathrm{O}$ abundance ratios are consistent with this pathlength dependence and they suggest an N/O source abundance close to $10 \%$ - larger than some previous estimates based on lower-energy isotope measurements (see e.g. [23]).

For this measurement, the dominant source of systematic error at high energy are uncertainties in the cross sections for producing secondaries by chargechanging interactions in the atmosphere above the instrument. Because there is a significant decrease in the interstellar pathlength with energy, the amount of boron, for example, at high energy is small - making the impact of uncertainties in the atmospheric boron contribution significant above $\sim 100 \mathrm{GeV} / \mathrm{n}$. At these energies the contribution from charge-changing interactions in the atmosphere is similar in size to the total production of boron during propagation through the Galaxy. As a consequence it seems likely that accurate measurements of $\mathrm{B} / \mathrm{C}$ on high-altitude balloon experiments will be ultimately limited by systematic errors in the $\mathrm{TeV} / \mathrm{n}$ region until knowledge of these atmospheric corrections can be improved. 


\section{Acknowledgements}

The work reported in this paper was funded by NASA research grants to the University of Maryland, the University of Chicago, Penn State University, and the Ohio State University, by the Korean Ministry of Science and Technology and the Creative Research Initiatives (MEMS Space Telescope) of MOST/KOSEF in Korea, and by INFN in Italy. The authors wish to acknowledge NASA/WFF for provision and operation of flight support systems; Art Ruitberg, Suong Le, and Curtis Dunsmore of NASA/GSFC, and Carlos Urdiales of Southwest Research Institute for assistance with HV design and potting; CERN for provision of excellent accelerator beams; the Fermi National Accelerator Lab Thin Films Group for high-quality polishing and aluminization of optical elements; and Columbia Scientific Ballooning Facility, National Science Foundations Office of Polar Programs, and Raytheon Polar Services Company for outstanding support of launch, flight and recovery operations in Antarctica. The TCD group acknowledges engineering contributions made by L. Engel, J. Passaneau and S. Posey. The TRD group acknowledges engineering contributions made by R. Northrop, C. Smith and G.A. Kelderhouse. The SCD group acknowledges contributions made by W. Han, H.J. Hyun, H.J. Kim, M.Y. Kim, K.W. Min, H. Park and K.I. Seon. 
Table 1

Table of measurements of Boron to Carbon Ratio

\begin{tabular}{|c|c|c|c|}
\hline $\begin{array}{c}\text { Kinetic Energy } \\
(\mathrm{GeV} / \mathrm{n})\end{array}$ & $\begin{array}{c}\text { Ratio B/C } \\
\text { measured }\end{array}$ & $\begin{array}{c}\text { Ratio B/C } \\
\text { corrected }\end{array}$ & $\begin{array}{c}\text { Energy Range } \\
(\mathrm{GeV} / \mathrm{n})\end{array}$ \\
\hline $1.4 \pm 0.2($ sys. $)$ & 0.353 & $0.320 \pm 0.007($ stat. $) \pm 0.03($ sys. $)$ & $1-4$ \\
$5.7 \pm 0.9($ sys. $)$ & 0.256 & $0.225 \pm 0.004($ stat. $) \pm 0.02($ sys. $)$ & $4-16$ \\
$23 \pm 3($ sys. $)$ & 0.185 & $0.155 \pm 0.005($ stat. $) \pm 0.014($ sys. $)$ & $16-63$ \\
$91 \pm 14($ sys. $)$ & 0.131 & $0.101 \pm 0.011($ stat. $) \pm 0.01($ sys. $)$ & $63-251$ \\
$363 \pm 54($ sys. $)$ & 0.099 & $0.071 \pm 0.025($ stat. $) \pm 0.01($ sys. $)$ & $251-1000$ \\
$1450 \pm 217($ sys. $)$ & 0.061 & $0.033 \pm 0.025($ stat. $) \pm 0.01($ sys. $)$ & $1000-4000$ \\
\hline
\end{tabular}

Table 2

Table of measurements of Nitrogen to Oxygen Ratio

\begin{tabular}{|c|c|c|c|}
\hline $\begin{array}{c}\text { Kinetic Energy } \\
(\mathrm{GeV} / \mathrm{n})\end{array}$ & $\begin{array}{c}\text { Ratio N/O } \\
\text { measured }\end{array}$ & $\begin{array}{c}\text { Ratio N/O } \\
\text { corrected }\end{array}$ & $\begin{array}{c}\text { Energy Range } \\
(\mathrm{GeV} / \mathrm{n})\end{array}$ \\
\hline $1.4 \pm 0.2($ sys. $)$ & 0.341 & $0.299 \pm 0.006($ stat. $) \pm 0.03($ sys. $)$ & $1-4$ \\
$5.7 \pm 0.9($ sys. $)$ & 0.286 & $0.246 \pm 0.004($ stat. $) \pm 0.025($ sys. $)$ & $4-16$ \\
$23 \pm 3($ sys. $)$ & 0.248 & $0.210 \pm 0.009($ stat. $) \pm 0.02($ sys. $)$ & $16-63$ \\
$91 \pm 14($ sys. $)$ & 0.211 & $0.174 \pm 0.026($ stat. $) \pm 0.02($ sys. $)$ & $63-251$ \\
$363 \pm 54($ sys. $)$ & 0.160 & $0.124 \pm 0.072($ stat. $) \pm 0.01($ sys. $)$ & $251-1000$ \\
$1450 \pm 217($ sys. $)$ & 0.083 & $0.050 \pm 0.034($ stat. $) \pm 0.01($ sys. $)$ & $1000-4000$ \\
\hline
\end{tabular}

Table 3

Table of measurements of Carbon to Oxygen Ratio

\begin{tabular}{|c|c|c|c|}
\hline $\begin{array}{c}\text { Kinetic Energy } \\
(\mathrm{GeV} / \mathrm{n})\end{array}$ & $\begin{array}{c}\text { Ratio C/O } \\
\text { measured }\end{array}$ & $\begin{array}{c}\text { Ratio C/O } \\
\text { corrected }\end{array}$ & $\begin{array}{c}\text { Energy Range } \\
(\mathrm{GeV} / \mathrm{n})\end{array}$ \\
\hline $1.4 \pm 0.2($ sys. $)$ & 1.19 & $1.10 \pm 0.01($ stat. $) \pm 0.1($ sys. $)$ & $1-4$ \\
$5.7 \pm 0.9($ sys. $)$ & 1.19 & $1.11 \pm 0.01($ stat. $) \pm 0.1($ sys. $)$ & $4-16$ \\
$23 \pm 3($ sys. $)$ & 1.25 & $1.16 \pm 0.03($ stat. $) \pm 0.1($ sys. $)$ & $16-63$ \\
$91 \pm 14($ sys. $)$ & 1.34 & $1.25 \pm 0.10($ stat. $) \pm 0.1($ sys. $)$ & $63-251$ \\
$363 \pm 54($ sys. $)$ & 1.35 & $1.25 \pm 0.32($ stat. $) \pm 0.1($ sys. $)$ & $251-1000$ \\
$1450 \pm 217($ sys. $)$ & 0.71 & $0.66 \pm 0.41($ stat. $) \pm 0.1($ sys. $)$ & $1000-4000$ \\
\hline
\end{tabular}




\section{References}

[1] A. W. Strong, I. V. Moskalenko, V. S. Ptuskin, Annual Review of Nuclear and Particle Science 57 (2007) 285-327.

[2] E. Juliusson, P. Meyer, D. Müller, Phys. Rev. Lett.29 (1972) 445-448.

[3] L. H. Smith, A. Buffington, D. F. Smoot, L. W. Alvarez, M. A. Wahlig, ApJ180 (1973) 987-1010.

[4] S. P. Swordy, D. Mueller, P. Meyer, J. L'Heureux, J. M. Grunsfeld, ApJ349 (1990) 625-633.

[5] J. J. Engelmann, P. Ferrando, A. Soutoul, P. Goret, E. Juliusson, A\&A233 (1990) 96-111.

[6] A. J. Davis, R. A. Mewaldt, W. R. Binns, E. R. Christian, A. C. Cummings, J. S. George, P. L. Hink, R. A. Leske, T. T. von Rosenvinge, M. E. Wiedenbeck, N. E. Yanasak, in: R. A. Mewaldt, J. R. Jokipii, M. A. Lee, E. Möbius, T. H. Zurbuchen (Eds.), Acceleration and Transport of Energetic Particles Observed in the Heliosphere, Vol. 528 of American Institute of Physics Conference Series, 2000, pp. 421-+.

[7] E.-S. Seo, et al., in: Proc. 29th ICRC (Pune), Vol. 3, 2005, pp. 101-104.

[8] H. S. Ahn, P. Allison, M. G. Bagliesi, J. J. Beatty, G. Bigongiari, P. Boyle, J. T. Childers, N. B. Conklin, S. Coutu, M. A. Duvernois, O. Ganel, J. H. Han, J. A. Jeon, K. C. Kim, J. K. Lee, M. H. Lee, L. Lutz, P. Maestro, A. Malinin, P. S. Marrocchesi, S. A. Minnick, S. I. Mognet, S. W. Nam, S. L. Nutter, I. H. Park, N. H. Park, E. S. Seo, R. Sina, S. P. Swordy, S. P. Wakely, J. Wu, J. Yang, Y. S. Yoon, R. Zei, S. Y. Zinn, Nuclear Instruments and Methods in Physics Research A 579 (2007) 1034-1053.

[9] H. S. Ahn, M. G. Bagliesi, J. J. Beatty, G. Bigongiari, A. Castellina, J. T. Childers, N. B. Conklin, S. Coutu, M. A. Duvernois, O. Ganel, J. H. Han, H. J. Hyun, T. G. Kang, H. J. Kim, K. C. Kim, M. Y. Kim, T. Kim, Y. J. Kim, J. K. Lee, M. H. Lee, L. Lutz, P. Maestro, A. Malinine, P. S. Marrocchesi, S. I. Mognet, S. W. Nam, S. Nutter, N. H. Park, H. Park, I. H. Park, E. S. Seo, R. Sina, S. Syed, C. Song, S. Swordy, J. Wu, J. Yang, H. Q. Zhang, R. Zei, S. Y. Zinn, Nuclear Physics B Proceedings Supplements 150 (2006) 272-275.

[10] I. H. Park, N. H. Park, S. W. Nam, et al., Nuclear Instruments and Methods in Physics Research A 570 (2007) 286-291.

[11] S. P. Wakely, APh 18 (2002) 67-87.

[12] A. H. Walenta, J. Fischer, H. Okuno, C. L. Wang, Nuclear Instruments and Methods 161 (1) (1979) 45-58.

[13] S. P. Swordy, P. Boyle, S. Wakely, in: Proc. 28th ICRC (Tskuba), 2003, pp. 2233-2236. 
[14] T. J. Brandt, et al., in: Proc. 30th ICRC (Merida), 2007.

[15] GEANT4 Collaboration, S. Agostinelli, J. Allison, K. Amako, J. Apostolakis, H. Araujo, P. Arce, M. Asai, D. Axen, S. Banerjee, G. Barrand, F. Behner, L. Bellagamba, J. Boudreau, L. Broglia, A. Brunengo, H. Burkhardt, S. Chauvie, J. Chuma, R. Chytracek, G. Cooperman, G. Cosmo, P. Degtyarenko, A. dell'Acqua, G. Depaola, D. Dietrich, R. Enami, A. Feliciello, C. Ferguson, H. Fesefeldt, G. Folger, F. Foppiano, A. Forti, S. Garelli, S. Giani, R. Giannitrapani, D. Gibin, J. J. Gómez Cadenas, I. González, G. Gracia Abril, G. Greeniaus, W. Greiner, V. Grichine, A. Grossheim, S. Guatelli, P. Gumplinger, R. Hamatsu, K. Hashimoto, H. Hasui, A. Heikkinen, A. Howard, V. Ivanchenko, A. Johnson, F. W. Jones, J. Kallenbach, N. Kanaya, M. Kawabata, Y. Kawabata, M. Kawaguti, S. Kelner, P. Kent, A. Kimura, T. Kodama, R. Kokoulin, M. Kossov, H. Kurashige, E. Lamanna, T. Lampén, V. Lara, V. Lefebure, F. Lei, M. Liendl, W. Lockman, F. Longo, S. Magni, M. Maire, E. Medernach, K. Minamimoto, P. Mora de Freitas, Y. Morita, K. Murakami, M. Nagamatu, R. Nartallo, P. Nieminen, T. Nishimura, K. Ohtsubo, M. Okamura, S. O'Neale, Y. Oohata, K. Paech, J. Perl, A. Pfeiffer, M. G. Pia, F. Ranjard, A. Rybin, S. Sadilov, E. di Salvo, G. Santin, T. Sasaki, N. Savvas, Y. Sawada, S. Scherer, S. Sei, V. Sirotenko, D. Smith, N. Starkov, H. Stoecker, J. Sulkimo, M. Takahata, S. Tanaka, E. Tcherniaev, E. Safai Tehrani, M. Tropeano, P. Truscott, H. Uno, L. Urban, P. Urban, M. Verderi, A. Walkden, W. Wander, H. Weber, J. P. Wellisch, T. Wenaus, D. C. Williams, D. Wright, T. Yamada, H. Yoshida, D. Zschiesche, Nuclear Instruments and Methods in Physics Research A 506 (2003) 250-303.

[16] P. Maestro, et al., in: Proc. 30th ICRC (Merida), 2007.

[17] E. Juliusson, ApJ191 (1974) 331-348.

[18] J. Buckley, J. Dwyer, D. Mueller, S. Swordy, K. K. Tang, ApJ429 (1994) 736747.

[19] C. H. Tsao, R. Silberberg, A. F. Barghouty, ApJ501 (1998) 920-+.

[20] J.-P. Meyer, ApJS57 (1985) 173-204.

[21] S. Swordy, in: Proc. 24th ICRC (Rome), 1995, p. 697.

[22] E. G. Berezhko, L. T. Ksenofontov, V. S. Ptuskin, V. N. Zirakashvili, H. J. Völk, A\&A410 (2003) 189-198.

[23] R. A. Mewaldt, J. D. Spalding, E. C. Stone, R. E. Vogt, ApJ251 (1981) L27-L31. 